\title{
KOOPERATIONEN VON UNIVERSITÄTSBIBLIOTHEKEN DER ÖFFENTLICHEN UNIVERSITÄTEN IN ÖSTERREICH IM RAHMEN DER HOCHSCHULRAUMSTRUIKTURMITTELPROJEKTE
}

\author{
von Bruno Bauer und Maria SeissI
}

Zusammenfassung: Die Finanzierung von innovativen Kooperationsprojekten der Universitätsbibliotheken an öffentlichen Universitäten aus zentralen Mitteln war lange Zeit ein Desiderat. Mit den Ausschreibungen der Hochschulraumstrukturmittelprojekte 2013 bzw. 2017 durch das für die Wissenschaft zuständige Bundesministerium wurden Anschubfinanzierungen für universitäre Kooperationsvorhaben, auch für Projekte der Verwaltung, bereitgestellt. Unter den vom Bundesministerium ausgewählten förderwürdigen Projektanträgen der Universitäten befinden sich auch fünf Projekte, die auf Initiative des Forums Universitätsbibliotheken Österreichs konzipiert wurden. Die Themenpalette reicht von Repositorien und Forschungsdaten über Open Access und Open Educational Resources bis zum Vergaberecht für wissenschaftliche Fachliteratur. Die bereits vorgelegten Ergebnisse und die noch umzusetzenden Vorhaben leisten einen wichtigen Beitrag dafür, dass die Informationsinfrastruktur in Österreich für Forschung und Lehre im europäischen und internationalen Kontext konkurrenzfähig und zukunftsfit weiterentwickelt werden kann.

Schlagwörter: Österreich; öffentliche Universität; Universitätsbibliothek; Kooperation; Hochschulraumstrukturmittelprojekt; e-Infrastructures Austria; E-Infrastructures Austria Plus; Vergaberecht; Austrian Transition to Open Access; Open Education Austria

\section{COOPERATIONS OF LIBRARIES OF PUBLIC UNIVERSITIES IN AUSTRIA IN THE CONTEXT OF THE HIGHER EDUCATION AREA STRUCTURAL FUNDING PROJECTS}

Abstract: For a long time, the funding of innovative cooperation projects of university libraries at public universities with central funds was a desideratum. Start-up financing for university cooperation projects, including administrative projects, was provided by the calls for proposals for the Higher Education Area Structural Funding projects in 2013 and 2017, respectively, by the Federal Ministry responsible for science. Among the projects that were selected by the Federal Ministry were also five designed on the initiative of the Forum Universitätsbibliotheken Österreichs. The topics range from repositories and research data to Open Access and Open Educational Resources to procurement law for scholarly literature. The results already presented and the projects still to be implemented make an important contribution to ensuring that the 
information infrastructure in Austria can be developed competitively and future-proof for research and teaching in a European and international context.

Keywords: Austria; public university; library; cooperation; Higher Education Area Structural Funding project; e-Infrastructures Austria; e-Infrastructures Austria Plus; procurement law; Austrian Transition to Open Access; Open Education Austria

DOI: https://doi.org/10.31263/voebm.v72i1.2280

(C) Bruno Bauer, Maria Seissl

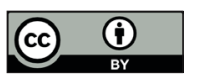

Dieses Werk ist lizenziert unter einer

Creative-Commons-Lizenz Namensnennung 4.0 International

\section{Inhalt}

1. Grundlegende Bemerkungen zu Kooperationen von Bibliotheken in Österreich

2. Ausschreibung von Hochschulraumstrukturmittelprojekten

3. e-Infrastructures Austria (2014-2016)

4. e-Infrastructures Austria Plus (2017-2019)

5. Entwicklung und Umsetzung eines Beschaffungsprozesses für österreichische Universitätsbibliotheken (2014-2016)

6. Austrian Transition to Open Access (2017-2020)

7. Open Education Austria (2017-2018)

8. Resümee

\section{Grundlegende Bemerkungen zu Kooperationen von Bibliotheken in Österreich}

Die wissenschaftlichen Bibliotheken in Österreich kooperieren traditionell sehr gut im Österreichischen Bibliothekenverbund (OBV), einem nationalen Verbund von universitären, wissenschaftlichen und administrativen Bibliotheken. Aktuell besteht der OBV aus 90 Bibliotheken und Informationseinrichtungen, darunter befınden sich die Österreichische Nationalbibliothek, die Bibliotheken von 21 öffentlichen Universitäten, 5 Privatuniversitäten, 12 Fachhochschulen und neun pädagogischen Hochschulen. Die seit 2002 eingerichtete Österreichische Bibliothekenverbund- und Service GmbH (OBVSG, https://www.obvsg.at/) fungiert als zentrale Servicestelle, die neben Services für die beteiligten Einrichtungen eine zentrale Bibliotheks- und Informationsinfrastruktur (Bibliotheksverbundsystem, Such- 
maschinentechnologie) betreibt. Die Basisfinanzierung der OBVSG für die Universitätsbibliotheken und die Österreichische Nationalbibliothek erfolgt durch das zuständige Bundesministerium.

Seit 2005 besteht eine intensive freiwillige Zusammenarbeit wissenschaftlicher Bibliotheken in der 2005 gegründeten Kooperation E-Medien Österreich (KEMÖ, https://www.konsortien.at/). Ziel der KEMÖ ist es, kostengünstig Datenbanken, elektronische Zeitschriften und elektronische Bücher gemeinschaftlich zu lizenzieren. Durch den konsortialen Erwerb im Rahmen von Bibliothekskonsortien besteht aufgrund der größeren Auftragsvolumina eine bessere Verhandlungsposition der beteiligten Bibliotheken gegenüber den Anbietern. Vorteile von Konsortien sind eine Minimierung des Verwaltungsaufwands durch Übertragung der Verhandlungsführung und Administration der Verträge an eine Geschäftsstelle sowie der Bezug von elektronischen Ressourcen zu kostengünstigeren Einkaufspreisen durch Rabattierungen. Erfolgte die Gründung der KEMÖ, deren Geschäftsstelle an der OBVSG angesiedelt ist, auf Initiative der Universitätsbibliotheken von 13 öffentlichen Universitäten, so ist die Zahl der Kooperationspartner auf 57 angestiegen, darunter befinden sich die Bibliotheken von 18 öffentlichen Universitäten, der Universität für Weiterbildung Krems, von 8 Privatuniversitäten, von 18 Fachhochschulen, von 12 weiteren wissenschaftlichen Einrichtungen sowie die Österreichische Nationalbibliothek. Das aktuelle Portfolio der KEMÖ umfasst zirka 70 Produktverträge.

Eine besonders wichtige Rolle in der österreichischen Bibliothekslandschaft kommt den 21 öffentlichen Universitäten zu: Karl-Franzens-Universität Graz, Medizinische Universität Graz, Technische Universität Graz, Universität für Musik und Darstellende Kunst Graz, Leopold-FranzensUniversität Innsbruck, Medizinische Universität Innsbruck, Alpen-AdriaUniversität Klagenfurt, Montantuniversität Leoben, Johannes-KeplerUniversität Linz, Universität für Künstlerische und Industrielle Gestaltung Linz, Paris-Lodron-Universität Salzburg, Universität Mozarteum Salzburg, Universität Wien, Medizinische Universität Wien, Technische Universität Wien, Universität für Bodenkultur, Veterinärmedizinische Universität Wien, Wirtschaftsuniversität Wien, Akademie der bildenden Künste Wien, Universität für Angewandte Kunst Wien, Universität für Musik und Darstellende Kunst Wien.

Mit der Implementierung des neuen Universitätsgesetzes wurden die öffentlichen Universitäten Österreichs in die Vollrechtsfähigkeit entlassen, dem Staat kommt weiterhin eine zentrale Rolle bei der Finanzierung der Universitäten zu. Wesentliche Elemente im Zusammenspiel zwischen den öffentlichen Universitäten und dem Bund sind die alle drei Jahre abzu- 
schließenden Leistungsvereinbarungen sowie die von den Universitäten jährlich vorzulegenden Wissensbilanzen.

Die Literaturversorgung der öffentlichen Universitäten ist Aufgabe der jeweiligen Universitätsbibliotheken, abgesehen von der 2004 neu errichteten Medizinischen Universität Innsbruck, an der keine eigenständige Universitätsbibliothek eingerichtet wurde. Die an der Universität Innsbruck etablierte Universitäts- und Landesbibliothek Tirol ist für die Literatur- und Informationsversorgung beider Innsbrucker Universitäten zuständig.

Kooperationsplattform für jene Universitätsbibliotheken, deren Universitäten auch in der Österreichischen Universitätenkonferenz vertreten sind, ist das Forum Universitätsbibliotheken Österreichs (ubifo, http://ubifo. at/). Das Forum kooperiert traditionell bei vielen Projekten, setzt laufend wichtige Impulse im österreichischen Bibliothekswesen und ist Träger wichtiger Initiativen für das wissenschaftliche Bibliothekswesen. So wurde auf Initiative des Forums die Kooperation E-Medien Österreich (KEMÖ) eingerichtet, die Österreichische Bibliotheksstatistik (ÖBS) etabliert und die Implementierung der aktuell eingesetzten Suchmaschinentechnologie sowie des neuen Bibliotheksverwaltungssystems forciert. Auch wichtige Studien zu Aspekten des Bibliotheks- und Informationswesens werden laufend vom Forum in Auftrag gegeben (Langzeitarchivierung, Pay-per-Use, Verteilte Archivierung, Open Access). ${ }^{1}$

Auch auf internationaler Ebene bestehen wichtige Kooperationen, an denen viele wissenschaftliche Bibliotheken in Österreich beteiligt sind.

Im Rahmen der Elektronischen Zeitschriftenbibliothek (EZB, http://rzblx1.uni-regensburg.de/ezeit/), einer kooperativ betriebenen Plattform für den schnellen, strukturierten und einheitlichen Zugriff auf ca. 98.000 Zeitschriften, beteiligen sich ca. 640 Bibliotheken, darunter 62 aus Österreich.

Das Datenbankinformationssystem (DBIS, http://rzblx10.uni-regensburg.de/dbinfo/), ein kooperatives Service für den Zugriff auf ca.13.000 wissenschaftliche Datenbanken, wird von ca. 330 Bibliotheken, davon 25 aus Österreich, genutzt.

An Subito - Dokumente aus Bibliotheken (http://www.subito-doc. de/), dem größten europäischen Literaturlieferdienst, der von 42 Lieferbibliotheken getragen wird, sind auch zwei österreichische Bibliotheken als Lieferbibliotheken beteiligt.

An E-books On Demand (EOD, http://books2ebooks.eu/de), einem europäischen Digitalisierungsnetzwerk mit der Zielsetzung, urheberrechtsfreie Werke aus der Zeit von 1500 bis 1900 als elektronische Bücher zugänglich zu machen, kooperieren 38 Bibliotheken aus 13 Ländern, darunter sieben österreichische Bibliotheken. 
An der Österreichischen Bibliotheksstatistik (ÖBS, http://www.hbznrw.de/angebote/oebs/), die sich auf die Infrastruktur der Deutschen Bibliotheksstatistik (DBS) stützt, was dank einer Kooperation der OBVSG mit dem Hochschulbibliothekszentrum des Landes Nordrhein-Westfalen (hbz) ermöglicht wurde, beteiligen sich 22 österreichische Bibliotheken.

Auch wenn wissenschaftliche Bibliotheken in Österreich in den genannten Themenfeldern sehr gut kooperieren, so bestand bis 2014 ein Desiderat hinsichtlich der Fördermöglichkeiten und Weiterentwicklungsmaßnahmen von gemeinsamer Bibliotheks- und Informationsinfrastruktur auf nationaler Ebene.

Während etwa die deutsche Schwerpunktinitiative „Digitale Information" mit ihren Aktionsfeldern Nationale Lizenzierung, Nationale HostingStrategie, Forschungsprimärdaten, Open Access, Rechtliche Rahmenbedingungen und Virtuelle Forschungsumgebung das Ziel verfolgte, „Wissenschaftlerinnen und Wissenschaftler mit der bestmöglichen Informationsinfrastruktur auszustatten, die sie für ihre Forschung brauchen", fehlten in Österreich Rahmenbedingungen für eine vergleichbare Initiative. ${ }^{2}$

\section{Ausschreibung von Hochschulraumstrukturmittelprojekten}

Mit der Bereitstellung sogenannter Hochschulraumstrukturmittel durch das Bundesministerium für Wissenschaft, Forschung und Wirtschaft (BMWFW) - ab 2018 Bundesministerium für Bildung, Wissenschaft und Forschung (BMBWF) - wurde bzw. wird das Ziel verfolgt, eine Anschubfinanzierung für universitäre Kooperationsvorhaben zu leisten.

2013 wurden vom BMWFW Ausschreibungen für Hochschulraumstrukturmittelprojekte veröffentlicht, in deren Rahmen eine Anschubfinanzierung für Kooperationsvorhaben der Lehre und Forschung / Entwicklung und Erschließung der Künste (48 Mio. Euro) bzw. der Verwaltung (15 Mio. Euro) für die Jahre 2014 bis 2016 bereitgestellt wurde. ${ }^{3}$ Auf Initiative des Forums Universitätsbibliotheken Österreich wurden zwei Projekte - „Entwicklung und Umsetzung eines Beschaffungsprozesses für österreichische Universitätsbibliotheken" sowie „e-Infrastructures Austria“ mit den Schwerpunkten Repositorien, Forschungsdatenmanagement und Wissensnetzwerk - von den jeweils interessierten Universitäten eingereicht. Aufgrund der Vergabeentscheidung des BMWFW wurde beiden Projekten eine Förderung zuteil.

Für die 2016 erfolgte Ausschreibung weiterer Hochschulraumstrukturmittelprojekte wurden vom BMWFW insgesamt 97,5 Mio. Euro als Anschubfinanzierung für Kooperationsprojekte für die Jahre 2017 bis 2020 
bereitgestellt. ${ }^{4}$ Davon entfielen 50 Mio. Euro auf Projekte der Verwaltung, 35 Mio. Euro auf Projekte der Lehre und 12,5 Mio. Euro auf Projekte zur Verwaltungsinnovation. ${ }^{5}$

Auf Initiative des Forums Universitätsbibliotheken wurden erneut zwei Projekte - „e-Infrastructures Austria Plus" unter Einbeziehung von weiteren Serviceeinrichtungen der interessierten Universitäten sowie „Austrian Transition to Open Access (AT2OA)“ - von den jeweils interessierten Universitäten eingereicht. Auf Initiative des Center for Teaching and Learning der Universität Wien wurde mit „Open Education Austria“ ein weiteres Kooperationsprojekt von vier Universitäten vorgeschlagen, in das neben den Bereichen Hochschuldidaktik/Neue Medien/OER und Zentrale Informatikdienste auch zwei Universitätsbibliotheken involviert sind. Alle drei genannten Projektanträge wurden im Rahmen des Vergabeverfahrens des BMWFW positiv beurteilt und in die Liste der zu fördernden Projekte aufgenommen.

\section{3. e-Infrastructures Austria (2014-2016)}

\section{e-infrastructures austria}

Das Hochschulraumstrukturmittelprojekt „eInfrastructures Austria" verfolgte während der dreijährigen Laufzeit (2014-2016) das Ziel, Repositorieninfrastrukturen koordiniert aufzubauen und kooperativ weiterzuentwickeln. ${ }^{6}$ Das Projekt wurde von 25 Projektpartnern betrieben; die Projektleitung lag bei der Universität Wien. An „e-Infrastructures Austria“ beteiligten sich auch eine Einrichtung als assoziierter Projektpartner sowie fünf weitere Institutionen mit Beobachterstatus.

Die Gesamtprojektkosten für „e-Infrastructures Austria“ betrugen 4,12 Mio. EUR (davon Fördervolumen durch das BMWFW: 1,37 Mio. EUR).

Im Rahmen von „e-Infrastructures Austria“ konnten laufend Fortschritte in der Umsetzung der drei Teilprojekte zu den Themen Repositorien, Forschungsdaten und Wissensnetzwerk verzeichnet ${ }^{7} 9$ und das Projekt Ende 2016 erfolgreich abgeschlossen werden.

In Teilprojekt A wurden an den 25 Partnereinrichtungen als In-KindLeistung Repositorien eigenständig aufgebaut bzw. befanden sich zum Zeitpunkt des Projektabschlusses in Planung oder Umsetzung. ${ }^{10}$

Bis zum Projektbeginn 2014 war das Thema Forschungsdatenmanagement an den meisten österreichischen Universitäten nicht oder nur kaum präsent. Deshalb wurden in Teilprojekt B wichtige Maßnahmen auf diesem Gebiet gesetzt. So erfolgte 2015 eine österreichweite Befragung von Forschenden über ihren Umgang mit Forschungsdaten. Die Umfrage bestand 
aus 20 Fragen in den Kategorien Datentypen und Formate, Datenarchivierung, -sicherung und -verlust, ethische und rechtliche Aspekte, Zugänglichkeit und Nachnutzung sowie Infrastruktur und Services. Zielgruppe der Befragung war das wissenschaftliche und künstlerisch-wissenschaftliche Personal aller 21 öffentlich-rechtlichen Universitäten sowie von drei außeruniversitären Forschungseinrichtungen in Österreich. Mit einer Beteiligung von 3.026 Forschenden erzielte die Umfrage eine Rücklaufquote von 9 Prozent. Nach der Auswertung der Umfrageergebnisse wurden diese in einem Report (print und online) in deutscher ${ }^{11} 12$ und englischer ${ }^{13}$ Sprache veröffentlicht und im Rahmen einer Pressekonferenz an der Universität Wien präsentiert.

Aufbauend auf den Umfrageergebnissen wurde von einer Expertlnnengruppe, bestehend aus Vertreterinnen und Vertretern der Hochschulleitungen, der Universitätsbibliotheken, der Zentralen Informatikdienste, der Forschungsservices, der Forschungsförderungsinstitutionen und des zuständigen Bundesministeriums, ein strategisches Konzept für den Umgang mit Forschungsdaten an den österreichischen Hochschulen und Forschungseinrichtungen erarbeitet und eine Muster-Policy für Forschungsdatenmanagement erstellt. An einzelnen Partnereinrichtungen erfolgte die Erprobung von Forschungsdatenmanagementplänen.

In Teilprojekt $C$ wurde das Ziel verfolgt, Fortbildung und Vernetzung für Forschungsdatenmanagement zu ermöglichen. Neben zahlreichen Workshops wurden diese Vorgaben von Teilprojekt $C$ vor allem durch ein viertägiges Seminar für den professionellen Umgang mit Forschungsdaten erreicht, das im Juni 2016 an der Universität Wien stattgefunden hat. Die Erkenntnisse und Erfahrungen aus diesem Seminar flossen in das Curriculum eines Zertifikatskurses „Data Librarian“ (https://www.postgraduatecenter.at/weiterbildungsprogramme/kommunikation-medien/data-librarian/) ein, der an der Universität Wien eingerichtet wurde.

Die Aktivitäten und Ergebnisse von „e-Infrastructures Austria“ wurden in einem umfangreichen Bericht dokumentiert.

Die Themen Repositorien und Forschungsdaten, zu denen zu Beginn des Projektes, nur wenig Wissen in der österreichischen Hochschul- und Forschungslandschaft vorhanden war, wurden im Projekt „e-Infrastructures Austria" erfolgreich bearbeitet und nachhaltig etabliert. Weil das Projekt in den drei Projektjahren sehr stark gewachsen ist, war eine gut abgestimmte Governance der Projektpartnereinrichtungen ein wichtiger Erfolgsfaktor. ${ }^{14}$ Als Kennzahlen für die Dimension des Projektes „e-Infrastructures Austria“, das einen sehr hohen Grad an Vernetzung im Inland und im Ausland erreicht hat, sind etwa 12 ständige Arbeitsgruppen, 23 Workshops und Ver- 
anstaltungen, 27 Vorträge und Präsentationen im Rahmen von nationalen und internationalen Tagungen und Kongressen, 15 Beiträge in nationalen und internationalen Fachzeitschriften sowie acht Generalversammlungen zu nennen. Insgesamt waren am Netzwerk mehr als 100 Personen beteiligt.

Zusammenfassend ist festzuhalten, dass das Projekt „e-Infrastructures Austria" eine sehr gute Basis für die weitere Bearbeitung des Themas Forschungsdaten und Forschungsdatenmanagement gelegt hat. ${ }^{15}$ Diese erfolgreiche Arbeit war Voraussetzung, dass vom BMWFW als unmittelbare Fortsetzung von „e-Infrastructures Austria“ das Folgeprojekt „e-Infrastructures Austria Plus" bewilligt wurde.

Interessierte Personen können sich über die Ergebnisse von „e-Infrastructures Austria" auf der Projektwebsite informieren: https://e-Infrastructures. univie.ac.at.

\section{4. e-Infrastructures Austria Plus (2017-2019)}

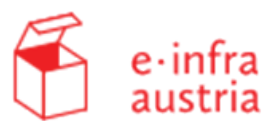

Im Zuge der vom BMWFW im Juli 2016 veröffentlichten Ausschreibung für die Vergabe von Hochschulraumstrukturmittel für den Bereich der Verwaltungsinnovation wurde unter Bezugnahme auf das Themenfeld „Open Innovation / Digital Roadmap / Open Access / Open Research Data" ein Folgeprojekt zu „e-Infrastructures Austria“ eingereicht, das auch bewilligt wurde. Das Projekt „e-Infrastructures Austria Plus" verfolgte während der dreijährigen Laufzeit (2017-2019) das Ziel, Infrastruktur für eScience in Österreich aufzubauen. Getragen wird das Projekt von acht öffentlichen Universitäten sowie einer Privatuniversität; die Projektleitung liegt bei der Universität Innsbruck. Weiters beteiligten sich sechs assoziierte Projektpartner an „e-Infrastructures Austria Plus".

Das Projekt gliedert sich in die sieben Arbeitspakete Research Lifecycle, Forschungsdatenmanagement Policy, Datenmanagement Pläne, institutionelle Repositorien, Metadaten, persistente Identifikatoren und Electronic Lab Notebook. Ergänzt werden die Arbeitspakete durch die Unterstützung der Initiative GO FAIR (Findable, Accessible, Interoperable, Reusable) und deren Ziele.

Im Rahmen von Arbeitspaket 1: „Research Lifecycle“ wurden an einigen Partnereinrichtungen Interviews mit Forschenden aus verschiedenen Fachbereichen über deren Umgang mit Forschungsdaten durchgeführt. Die Ergebnisse dieser Interviews, in denen Forschende ihren praktischen Umgang, ihre Erfahrungen und Vorstellungen zum Forschungsdatenma- 
nagement darstellen konnten, bilden die Grundlage für die weitere Ausgestaltung der Angebote für Forschende an den einzelnen Universitäten. In Arbeitspaket 2: „Forschungsdatenmanagement Policy“ wurden 21 Workshops mit der Zielsetzung der Implementierung von Policies anhand einer Muster Policy an den Partnereinrichtungen durchgeführt. Bisher wurden bereits an drei Universitäten Forschungsdatenmanagement Policies veröffentlicht (Universität für Musik und darstellende Kunst Wien, Medizinische Universität Wien, Technische Universität Wien), an neun weiteren Einrichtungen wird an Policies gearbeitet. In Arbeitspaket 3: „Datenmanagement-Pläne“ werden lokale Kompetenzen zu Datenmanagementplänen entwickelt, wobei ein Fokus auf der Open Data Policy des FWF inklusive eines verpflichtenden DMPs gelegt wird. In Arbeitspaket 4: „Institutionelle Repositorien“ liegt der Fokus auf dem von der Universität Innsbruck auf Basis einer Matrix der Research Data Alliance (RDA) gestarteten Auswahlprozesses für ein institutionelles Repositorium. Arbeitspaket 5: „Metadaten“ beschäftigt sich mit der automatischen Übernahme technischer Metadaten. In Arbeitspaket 6: „Persistente Identifikatoren" wird an der Erstellung einer DOI-Muster-Policy gearbeitet, die als Vorlage für eine institutionelle DOI-Policy herangezogen werden kann. In Arbeitspaket 7: „Electronic Lab Notebooks“ werden im Rahmen des Projektes Anforderungen von Wissenschaftlerinnen und Wissenschaftlern und der verschiedenen Fachbereiche gesammelt und analysiert, um damit eine Grundlage für die Auswahl und Einführung von Systemen an den Partnerinstitutionen oder in einem weiteren gemeinsamen Projekt zu schaffen.

Ein weiteres Ziel von „e-Infrastructures Austria Plus“ ist es, dazu beizutragen, dass GO FAIR an Forschungsinstitutionen in Österreich auf lokaler Ebene durch die Etablierung von Reference Points, den Knowhow-Transfer beim Aufbau von Infrastrukturen und forschungsunterstützenden Services sowie die Beratung von in diesem Bereich tätigen Arbeitsgruppen verankert wird.

Interessierte Personen können sich über den Status von „e-Infrastructures Austria Plus" mittels Newsletter ${ }^{16}$ bzw. der Projektwebsite informieren: https://www.e-Infrastructures.at.

\section{Entwicklung und Umsetzung eines Beschaffungsprozesses für öster- reichische Universitätsbibliotheken (2014-2016)}

Das Hochschulraumstrukturmittelprojekt „Entwicklung und Umsetzung eines Beschaffungsprozesses für österreichische Universitätsbibliotheken" verfolgte wäh- 
rend der dreijährigen Laufzeit (2014-2016) das Ziel, Vorarbeiten für Ausschreibungsverfahren für Literatur und Informationsressourcen unter juristischer Begleitung gemeinschaftlich zu erarbeiten. ${ }^{17}$ An diesem Projekt waren 16 Universitätsbibliotheken beteiligt (G16); die Projektleitung lag bei der Universität Wien.

Die Gesamtprojektkosten für das Projekt „Entwicklung und Umsetzung eines Beschaffungsprozesses für österreichische Universitätsbibliotheken" betrugen 0,45 Mio. EUR (davon Fördervolumen durch das BMWFW: 0,15 Mio. EUR).

In insgesamt fünf Arbeitsgruppen wurden die unterschiedlichen Anforderungen für die Produktgruppen Print-Bücher, Print-Zeitschriften, EBooks, E-Journals und Datenbanklizenzen ermittelt und profunde Unterlagen für die Abwicklung der jeweiligen Vergabeverfahren, insbesondere auch spezifische Leistungsverzeichnisse erstellt.

Der Fokus des Projektes lag zunächst auf der lokalen Beschaffung; eingebunden wurde aber auch die Geschäftsstelle der Kooperation E-Medien Österreich (KEMÖ), um die laufenden Vergabeverfahren entsprechend zu optimieren.

Die Arbeit und die Ergebnisse des Projektes wurden in einem internen Projekt-Wiki abgelegt; allen Partnereinrichtungen wurde zum Projektabschluss ein umfassender Bericht inklusive aller Unterlagen als ZIP-File zur Verfügung gestellt. Dieser gliedert sich in die Teilberichte für Print-Bücher, Print-Zeitschriften, E-Books, E-Journals und Datenbanklizenzen. In den einzelnen Abschnitten finden sich grundsätzliche Ausführungen zu den jeweiligen Produktgruppen (inklusive Besonderheiten und Abgrenzung, Definitionen), zum jeweils möglichen Beschaffungsprozess, zum Ablauf des jeweiligen Vergabeverfahrens sowie diverse Anhänge. Ergänzt wurde der Bericht um eine Dokumentation zur konsortialen Beschaffung, die von einer Arbeitsgruppe zur Umsetzung des Vergaberechts in der KEMÖ erstellt wurde.

\section{Austrian Transition to Open Access (2017-2020)}

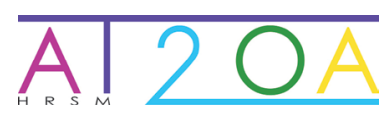

In der im Juli 2016 vom zuständigen Bundesministerium veröffentlichten Ausschreibung für die Vergabe von Hochschulraumstrukturmitteln für den Bereich der Verwaltungsinnovation wurde ein Schwerpunkt auf das Themenfeld „Open Innovation / Digital Roadmap / Open Access / Open Research Data“ gelegt: „Eingereichte Projektanträge zu Open Access sollen sich an den Empfehlungen des Open Access Network Austria (OANA) orientieren, nach denen 
die gesamte wissenschaftliche Publikationstätigkeit in Österreich bis 2025 auf Open Access umgestellt werden soll." 18

Im Herbst 2016 wurde das von den 21 öffentlichen Universitäten eingereichte Hochschulraumstrukturmittelprojekt „Austrian Transition to Open Access (AT2OA)“, in dem sieben der 16 „Empfehlungen für die Umsetzung von Open Access in Österreich"19 aufgegriffen worden waren, vom Bundesministerium bewilligt; die Projektleitung liegt bei der Universität Wien. Drei weitere Institutionen sind als kooptierte Partnereinrichtungen am Projekt beteiligt. $^{20}$

Die Gesamtprojektkosten für das Projekt „Austrian Transition to Open Access (AT2OA)“ betragen 10,57 Mio. EUR (davon Fördervolumen durch das BMWFW: 3,16 Mio. EUR).

AT2OA verfolgt während der vierjährigen Laufzeit (2017-2020) das Ziel, Open Access in Österreich durch geeignete, gemeinsam abgestimmte Maßnahmen voranzubringen und gliedert sich in vier Teilprojekte. In Teilprojekt 1 „Analyse der Auswirkung einer Umstellung auf Open Access" werden die finanziellen Auswirkungen einer vollständigen Transformation zu Open Access für die am Projekt beteiligten Einrichtungen analysiert. In Teilprojekt 2 „Finanzierung von Open Access-Übergangsmodellen“ steht die Erweiterung von subskriptionsbasierten Lizenzverträgen um eine Open Access-Komponente auf der Agenda. Der „Auf-, Ausbau und Finanzierung von Open Access-Publikationsfonds" ist Ziel von Teilprojekt 3. Teilprojekt 4 dient der „Förderung von OA-Publikationen und alternativen OA-Publikationsmodellen von Universitäten". ${ }^{21}$

Zur Hälfte des Projektzeitraums konnten bereits wichtige Teilziele von AT2OA erreicht werden. So liegt bereits der Abschlussbericht zur Transition-Studie vor ${ }^{22}{ }^{23}$ (Teilprojekt 1), eine „Leitlinie für Verlagsverträge für die OA-Transformation im Rahmen von HRSM-AT2OA“ und ein darauf aufsetzender richtungsweisender transformativer Lizenzvertrag mit Open Access-Komponente mit dem Verlag Wiley konnten abgeschlossen werden ${ }^{24}$ (Teilprojekt 2), Richtlinien für die Förderung von Open Access im Rahmen von Publikationsfonds ${ }^{25}$ (Teilprojekt 3 ) sowie „Empfehlungen für die Förderung von alternativen, nichtkommerziellen Open Science-Infrastrukturen \& -services (OSIS) durch Forschungseinrichtungen in Österreich" 26 (Teilprojekt 4) wurden vorgelegt.

Interessierte Personen können sich über den Status von AT2OA auf der Projektwebsite informieren: https://www.at2oa.at 


\section{Open Education Austria (2017-2018)}
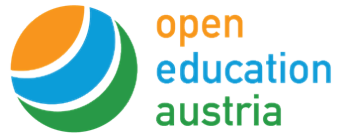

Im Rahmen des Hochschulraumstrukturmittelprojektes „Open Education Austria“ verfolgten Universitäten in Österreich während der zweijährigen Laufzeit (2017-2018) das Ziel, eine nationale Infrastruktur für Open Educational Resources (OER) zu entwickeln. E-Learning-Zentren, Zentrale Informatikdienste und Universitätsbibliotheken kooperierten, um Lehrende bei der Erstellung von OER-Materialien für Selbststudium und Lehre zu unterstützen. Das Projekt sollte auch zu einer schrittweisen Qualitätssteigerung im Lehren und Lernen führen und Good Practice-Materialien innerhalb der Fachcommunity besser sichtbar machen.

Erstellt wurden unter anderem mehr als 400 neue offene Bildungsressourcen, ein "Leitfaden für die Erstellung von Open Educational Resources“27 28, ein Massive Open Online Course (MOOC) sowie ein Prototyp für ein Fachportal zu OER.

An „Open Education Austria“ waren vier Universitäten beteiligt; die Projektleitung lag bei der Universität Wien. Mitgearbeitet haben Vertreterinnen und Vertreter aus den Bereichen Hochschuldidaktik/Neue Medien/ OER, Universitätsbibliothek sowie Zentraler Informatikdienst.

\section{Resümee}

Auf Basis der vom Bundesministerium in den Ausschreibungen 2013 bzw. 2016 bereitgestellten Anschubfinanzierung wurden von den Universitätsbibliotheken seit 2014 wichtige Kooperationsvorhaben realisiert bzw. befinden sich derzeit in Umsetzung.

Im Rahmen des Projektes „E-Infrastructures Austria“ konnten die angestrebten Ziele - Aufbau von Repositorien (Teilprojekt A), grundlegende Schritte zum Thema Forschungsdatenmanagement (Teilprojekt B) sowie Vernetzung und Fortbildung (Teilprojekt C) - erreicht werden. Von den Universitätsbibliotheken als Initiatorinnen des Projektes wurden während der Projektlaufzeit 2014 bis 2016 auch die Zentralen Informatikdienste, die Forschungsservices und Rechtsabteilungen sowie die wissenschaftliche Community für die Mitarbeit am Projekt gewonnen.

Die erfolgreiche Arbeit von "e-Infrastructures Austria" schuf eine ausgezeichnete Voraussetzung für das Folgeprojekt „e-Infrastructures Austria Plus“, in dessen Rahmen zwischen 2017 und 2019 die Arbeitspakete Research 
Lifecycle, Forschungsdatenmanagement Policy, Datenmanagement Pläne, institutionelle Repositorien, Metadaten, persistente Identifikatoren und Electronic Lab Notebook bearbeitet sowie die Initiative GO FAIR unterstützt werden. Damit werden wichtige Grundlagen geschaffen, um die österreichischen Universitäten und ihre Forschenden fit für die European Open Science Cloud zu machen.

Im Rahmen des Projektes „Entwicklung und Umsetzung eines Beschaffungsprozesses für österreichische Universitätsbibliotheken" wurde zwischen 2014 und 2016 gemeinschaftlich von den beteiligten Universitätsbibliotheken und unter juristischer Begleitung Ausschreibungsunterlagen für Literatur und Informationsressourcen erstellt, die für aktuelle Vergabeverfahren an den einzelnen beteiligten Institutionen und auch in der Kooperation E-Medien Österreich genutzt werden.

Das Projekt „Austrian Transition to Open Access“, das zwischen 2017 und 2020 Open Access in Österreich durch geeignete, gemeinsam abgestimmte Maßnahmen voranbringen soll, konnte mit der Transition-Studie, in der der Finanzierungsbedarf der einzelnen Partnerinstitutionen während der Umstellungsphase des Publikationswesens von Closed zu Open Access errechnet wurde, und mit dem richtungsweisenden transformativen Lizenzvertrag mit Open Access-Komponente mit dem Verlag Wiley bereits wesentliche Ziele in einzelnen Teilprojekten umsetzen.

Im Rahmen des Projektes „Open Education Austria“ wurde zwischen 2017 und 2018 eine nationale Infrastruktur für Open Educational Resources (OER) in Kooperation von E-Learning-Zentren, Zentralen Informatikdiensten und Universitätsbibliotheken entwickelt.

Dank der Hochschulraumstrukturmittel, die vom zuständigen Bundesministerium seit 2014 (und noch bis 2020) als Anschubfinanzierung für Kooperationsprojekte der Universitäten bereitgestellt werden, konnten wichtige und innovative Themen der Literatur- und Informationsversorgung auf Initiative der Universitätsbibliotheken - von Repositorien und Forschungsdaten über Open Access und Open Educational Resources bis zum Vergaberecht - unter optimalen Rahmenbedingungen gemeinschaftlich und strukturiert bearbeitet und umgesetzt werden.

Auf die Bedeutung der Hochschulraumstrukturmittelprojekte wurde 2018 auch vom österreichischen Bundesminister Heinz Faßmann in einem "Vortrag an den Ministerrat" hingewiesen, in dem die „Vorhaben des Bundesministers für Bildung, Wissenschaft und Forschung zur ZUKUNFT DIGITALISIERUNG - Die österreichischen Universitäten am Weg ins digitale Zeitalter" vorgestellt wurden. ${ }^{29}$ Unter dem Punkt „Aktuelle Strategien, Initiativen und Fördermaßnahmen" wurden die Hochschulraumstrukturmittel-Projekte „e-Infrastructures 
Austria“, „E-Infrastructures Austria Plus", „Austrian Transition to Open Access (AT2OA)“, „Open Education Austria“ sowie „Portfolio/Showroom - Making Art Research Accessible", ein Projekt unter der Leitung der Universität für angewandte Kunst Wien ${ }^{30}$, angeführt. Unter dem Punkt „Strategien und Ziele für die künftigen Entwicklungen" wird in drei der sechs angeführten Aktionsfelder ( „Institutionelle Digitalisierungsstrategie“, „Einschlägige Ausschreibung „Digitalisierung" des BMBWF“, „Weiterentwicklung von Open Science“) auf die Hochschulraumstrukturmittelprojekte Bezug genommen. Als wesentliche Maßnahme wird u.a. eine Ausschreibung „Digitalisierung" im Rahmen der Universitätsfinanzierung der Leistungsvereinbarungsperiode 2019-2021 (ähnlich den HRSM-Ausschreibungen) genannt, damit „die laufenden kooperativen HRSMProjekte [...] nachhaltig in die künftigen strategischen Entwicklungen eingebettet werden“. Als weitere wesentliche Maßnahme angeführt wird die „Nutzung der Leistungsvereinbarungen mit den Universitäten und Forschungsstätten um einerseits die Outcomes der HRSM-Projekte auf institutioneller Ebene umzusetzen und andererseits das Ziel zu erreichen, die einzelnen Maßnahmen zu einer kooperativen Open Science Landschaft zu verbinden".

Somit ist festzuhalten, dass mit den bereits vorgelegten Ergebnissen und den noch umzusetzenden Vorhaben aus den von den Universitätsbibliotheken betriebenen Hochschulraumstrukturmittelprojekten Grundlagen geschaffen wurden und werden, um die Informationsinfrastruktur in Österreich für Forschung und Lehre im europäischen und internationalen Kontext konkurrenzfähig und zukunftsfit weiterentwickeln zu können.

Mag. Bruno Bauer

ORCID: https://orcid.org/0000-0002-4729-331X Medizinische Universität Wien, Universitätsbibliothek E-Mail: bruno.bauer@meduniwien.ac.at

Mag. ${ }^{a}$ Maria Seissl Universität Wien, Bibliotheks- und Archivwesen E-Mail: maria.seissl@univie.ac.at 
1 Bauer, Bruno; Schiller, Robert: Das Forum der Universitätsbibliotheken Österreichs (ubifo) und seine Vorgängergremien. Geschichte und Entwicklung der Kooperationen der Universitätsbibliotheken. In: Niedermair, Klaus; Schuler, Dietmar (Hrsg.): Die Bibliothek in der Zukunft: regional - global: Lesen, Studieren und Forschen im Wandel. Festschrift für Hofrat Dr. Martin Wieser anlässlich seiner Versetzung in den Ruhestand. Innsbruck: Innsbruck Univ. Press, 2015, S. 57-71. Online unter: https://ubifo.at/images/WIESER_FESTSCHRIFT_2014_06-Bauer_ Bruno_Schiller_Robert_Das_Forum_der_Universitaetsbibliotheken_ Oesterreichs.pdf

2 Bauer, Bruno: Braucht Österreich eine Schwerpunktinitiative „Digitale Information"? Status quo und Perspektiven für die Hochschulbibliotheken. In: Mitteilungen der Vereinigung Österreichischer Bibliothekarinnen und Bibliothekare 65 (2012), Nr. 3/4, S. 394-415. Online unter: http:// eprints.rclis.org/18463/

3 BMWF: Hochschulraum-Strukturmittel - Karlheinz Töchterle: 48 Millionen Euro für gezielte Kooperationen in Lehre und Forschung. In: LISA vienna / life science austria, 17.09.2013. Online unter: https://www. lisavienna.at/de/news/bmwf-hochschulraum-strukturmittel-karlheinztoechterle-48-millionen-euro-fuer-gezielte-kooperation/

4 BMWFW: 750 Millionen für strategische Unientwicklung. Hochschulraumstrukturmittel steigen um 66 Prozent - Wissenstransfer, Studierende und Lehrerbildung als Schwerpunkte. In: Austria Innovativ. Online unter: https://www.austriainnovativ.at/story/article/bmwfw-750-millionen-fuer-strategische-unientwicklung/

5 Mitterlehner: Universitäts-Administration moderner und effizienter gestalten. Wissenschaftsministerium startet dritte Ausschreibung für Hochschulraumstrukturmittel - 12,5 Millionen Euro für Verwaltungsprojekte. In: OTS0010 5 II 0439 MWA0002 XI, 19.07.2016. Online unter: https://www.ots.at/presseaussendung/OTS_20160719_ OTS0010/mitterlehner-universitaets-administration-moderner-undeffizienter-gestalten

6 Sanchéz Solís, Barbara: e-Infrastructures Austria. In: Mitteilungen der Vereinigung Österreichischer Bibliothekarinnen und Bibliothekare 67 (2014) Nr. 2, S. 195-204. Online unter: http://eprints.rclis.org/23845/

7 Bauer, Bruno; Budroni, Paolo; Ferus, Andreas; Ganguly, Raman; Ramminger, Eva; Sánchez Solís, Barbara: e-Infrastructures Austria 2014: Bericht über das erste Jahr des Hochschulraumstrukturmittelprojekts für den koordinierten Aufbau und die kooperative Weiterentwicklung von Repositorieninfrastrukturen. In: Mitteilungen der Vereinigung Österrei- 
chischer Bibliothekarinnen und Bibliothekare 68 (2015), Nr. 1, S. 91-118. https://doi.org/10.31263/voebm.v68i1.1000

8 Bauer, Bruno; Budroni, Paolo; Ferus, Andreas; Ganguly, Raman; Ramminger, Eva; Sánchez Solís, Barbara: e-Infrastructures Austria 2015: Bericht über das zweite Jahr des Hochschulraumstrukturmittelprojekts für den koordinierten Aufbau und die kooperative Weiterentwicklung von Repositorieninfrastrukturen. In: Mitteilungen der Vereinigung Österreichischer Bibliothekarinnen und Bibliothekare, 69 (2016), Nr. 1, S. 10-40. https://doi.org/10.31263/voebm.v69i1.1394

9 Bauer, Bruno; Budroni, Paolo; Ferus, Andreas; Ganguly, Raman; Ramminger, Eva; Sánchez Solís, Barbara: e-Infrastructures Austria 2016: Bericht über das dritte Jahr des Hochschulraumstrukturmittelprojekts für den koordinierten Aufbau und die kooperative Weiterentwicklung von Repositorieninfrastrukturen. In: Mitteilungen der Vereinigung Österreichischer Bibliothekarinnen und Bibliothekare 70 (2017), Nr. 1, S. 66-93. https://doi.org/10.31263/voebm.v70i1.1834

10 Bauer, Bruno; Ferus, Andreas: Österreichische Repositorien in OpenDOAR und re3data.org: Entwicklung und Status von Infrastrukturen für Green Open Access und Forschungsdaten. In: Mitteilungen der Vereinigung Österreichischer Bibliothekarinnen und Bibliothekare 71 (2018), Nr. 1, S. 70-86. https://doi.org/10.31263/voebm.v71i1.2037

11 Bauer, Bruno; Ferus, Andreas; Gorraiz, Juan; Gründhammer, Veronika; Gumpenberger, Christian; Maly, Nikolaus; Mühlegger, Johannes Michael; Preza, José Luis; Sanchéz Solís, Barbara; Schmidt, Nora; Steineder, Christian: Forschende und ihre Daten. Ergebnisse einer österreichweiten Befragung - Report 2015. Version 1.2. https://doi.org.10.5281/ zenodo.32043

12 Bauer, Bruno; Ferus, Andreas; Gorraiz, Juan; Gründhammer, Veronika; Gumpenberger, Christian; Maly, Nikolaus; Mühlegger, Johannes Michael; Preza, José Luis; Sanchéz Solís, Barbara; Schmidt, Nora; Steineder, Christian: Forschende und ihre Daten: Ergebnisse einer österreichweiten Befragung. Report 2015 - Executive Summary und Empfehlungen. Mitteilungen der Vereinigung Österreichischer Bibliothekarinnen und Bibliothekare 68 (2015), Nr. 3/4, S. 515-579. https://doi.org/10.31263/ voebm.v68i3.1298

13 Bauer, Bruno; Ferus, Andreas; Gorraiz, Juan; Gründhammer, Veronika; Gumpenberger, Christian; Maly, Nikolaus; Mühlegger, Johannes Michael; Preza, José Luis; Sanchéz Solís, Barbara; Schmidt, Nora; Steineder, Christian: Researchers and their data. Results of an Austrian Survey Report 2015. Version 1.2. https://doi.org/10.5281/zenodo.34005 
14 Budroni, Paolo: Good Governance. Strukturen zur Sicherung der Nachhaltigkeit von Projekten im akademischen Umfeld. Das nationale Projekt e-Infrastructures Austria. In: ABI Technik 36 (2016), Nr. 1, S. 24-36. https://doi.org/10.1515/abitech-2016-0002

15 e-Infrastructures Austria (Hrsg.): Projektbericht 2014-2016, Version 2.0. Wien, Jänner 2017. Online unter: https://phaidra.univie.ac.at/detail_object/o:460779

16 E-Infrastructures Austria Plus: Newsletter Februar 2019. Online unter: https://www.e-Infrastructures.at/de/e-infra-newsletter-2019

17 Bauer, Bruno: Aktuelle Entwicklungen an den österreichischen Bibliotheken 2013. In: Bibliothek, Forschung und Praxis 38 (2014), Nr. 2, S. 272 282. https://doi.org/10.1515/bfp-2014-0042

18 Bauer, Bruno: Open Access in Österreich (4a). In: Söllner, Konstanze; Mittermaier, Bernhard (Hrsg.): Praxishandbuch Open Access. Berlin, Boston: de Gruyter, 2017, S. 207-214. (De Gruyter Praxishandbuch). https://doi.org/10.1515/9783110494068-024

19 Bauer, Bruno; Blechl, Guido; Bock, Christoph; Danowski, Patrick; Ferus, Andreas; Graschopf, Anton; König, Thomas; Mayer, Katja; Nentwich, Michael; Reckling, Falk; Rieck, Katharina; Seitz, Peter; Stöger, Herwig; Welzig, Elvira: Empfehlungen für die Umsetzung von Open Access in Österreich. In: Mitteilungen der Vereinigung Österreichischer Bibliothekarinnen und Bibliothekare 68 (2016), Nr. 3/4, S. 580-607. https://doi. org/10.31263/voebm.v68i3.1299

20 Bauer, Bruno: Aktuelle Entwicklungen an den österreichischen Bibliotheken 2016. In: Bibliothek, Forschung und Praxis 42 (2018), Nr. 1, S. 141153. https://doi.org/10.1515/bfp-2018-0018

21 Bauer, Bruno; Capellaro, Christof; Ferus, Andreas; Fessler, Georg; Granat, Renata; Hölbling, Lothar; Kaier, Christian; Koren-Wilhelmer, Frank; Kromp, Brigitte; Seissl Maria; Zarka, Tobias: Austrian Transition to Open Access (AT2OA). In: Bibliothek Forschung und Praxis 42 (2018), Nr. 3, S. 463-475. https://doi.org/10.1515/bfp-2018-0062

22 Fessler, Georg; Hölbling, Lothar: Ausbau von Open Access an den österreichischen Universitäten: Budgetärer Mehrbedarf für die Jahre 2019-2021. Wien: AT2OA, 2019. Zenodo. https://doi.org/10.5281/ zenodo. 2621015

23 Fessler, Georg: Ausbau von Open Access an den österreichischen Universitäten: budgetärer Mehrbedarf für die Jahre 2019-2021. Zusammenfassung des HRSM AT2OA Transition-Studien-Abschlussberichts. In: Mitteilungen der Vereinigung Österreichischer Bibliothekarinnen und Bibliothekare 72 (2019), Nr. 1, S. 35-49. https://doi.org/10.31263/voebm.v72i1.2275 
24 Kromp, Brigitte; Koren-Wilhelmer, Frank: Finanzierung von Open Access-Übergangsmodellen: Leitlinie für Verlagsverträge für die Open Access Transformation. In: Mitteilungen der Vereinigung Österreichischer Bibliothekarinnen und Bibliothekare 72 (2019), Nr. 1, S. 66-73. https://doi. org/10.31263/voebm.v72i1.2277

25 Capellaro, Christof; Kaier, Christian: Förderbedingungen für Publikationsfonds: Open Access - ganz oder gar nicht. In: Mitteilungen der Vereinigung Österreichischer Bibliothekarinnen und Bibliothekare 72 (2019), Nr. 1, S. 74-88. https://doi.org/10.31263/voebm.v72i1.2278

26 Ferus, Andreas; Reckling, Falk: Die Förderung von alternativen, nichtkommerziellen Open Science-Infrastrukturen \& -services (OSIS) durch Forschungseinrichtungen in Österreich: Empfehlungen, Kriterien \& Modelle. In: Mitteilungen der Vereinigung Österreichischer Bibliothekarinnen und Bibliothekare 72 (2019), Nr. 1, S. 89-105. https://doi.org/10.31263/ voebm.v72i1.2279

27 Zimmermann, Claudia: Leitfaden für die Erstellung von Open Educational Resources. Informationen und praktische Übungen für Hochschullehrende. Hrsg. von Open Education Austria, Graz 2018. Online unter: https://openeducation.at/fileadmin/user_upload/p_oea/OEALeitfaden_online_Aufl2.pdf

28 Zimmermann, Claudia: Guideline for the Creation of Open Educational Resources. Information and Practical Exercises for Lecturers in Higher Education. Open Education Austria, Graz 2018. Online unter: https:// openeducation.at/fileadmin/user_upload/P_oea/OEA-Guideline_online_final_english.pdf

29 Faßmann, Heinz: Vorhaben des Bundesministers für Bildung, Wissenschaft und Forschung zur ZUKUNFT DIGITALISIERUNG - Die österreichischen Universitäten am Weg ins digitale Zeitalter. Vortrag an den Ministerrat, 07.06.2018. Online unter: https://www.bundeskanzleramt.gv.at/documents/131008/877075/21_9_mrv.pdf/1e321 eea066c-4d71-8143-b757d21cfe21

30 Bettel, Florian; Frank, Alexandra; Miljes, Wiebke: Sichtbarkeit, Sicherheit, Usability und Weiterverwendung - Benutzer/innenorientierte FIS/ CRIS-Entwicklung am Beispiel von „Portfolio/Showroom“. In: Mitteilungen der Vereinigung Österreichischer Bibliothekarinnen und Bibliothekare 71 (2018), Nr. 1, S. 136-148. https://doi.org/10.31263/voebm. v71i1.1989 